\section{Rat reality show blurs quality control}

The governance of science, including the whole system of quality assurance, depends on specialist access to resources and publication. A new and radical engagement of the public in reality and crowd-sourced science is calling this principle into question.

Following the furore surrounding cancer claims for herbicide-resistant transgenic maize (Nature 489, 484; 2012; and Nature 492, 12; 2012), Russian scientists intend to run a 'rat reality show' that will be broadcast over the Internet around the clock. This year-long, real-time feeding experiment aims to test the safety of genetically modified food products (see go.nature.com/ qkvzqe). Actually witnessing the experiment, the team suggests, will allow the public to draw their own conclusions. If the scientists are unable to fund the show from orthodox sources, they plan to crowd-source public funding.

It would be easy to dismiss such 'reality' experiments as a stunt - as frivolity leading to demagoguery. But social media are increasingly influencing mainstream scientific communication and could stimulate a spread in reality science, blurring the demarcations on which the legitimacy and quality assurance of science traditionally depend.

Jerome R. Ravetz, Peter Healey, Steve Rayner Institute for Science, Innovation and Society, University of Oxford, UK. steve.rayner@insis.ox.ac.uk

\section{Cancer goal: deadline will not erode trust}

We at the US National Breast Cancer Coalition (NBCC) disagree with your assessment of our 2020 goal of knowing how to prevent breast cancer and its associated deaths (Nature 491, $637 ; 2012$ ). Working towards that

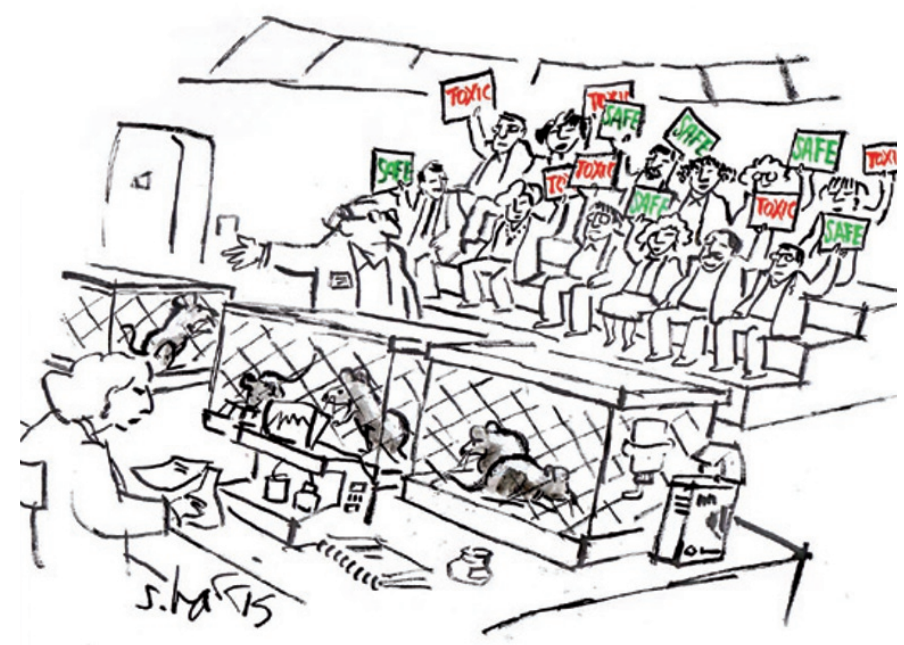

goal will not erode public trust, as you suggest.

Nearly 500,000 women worldwide died of breast cancer last year, despite billions of dollars being invested in research. Many scientists believe that current funding systems favour 'safe' research over bold new ideas. As a result, progress is incremental, leading to slightly better treatments, surgical interventions and radiation regimes. These may provide some benefit, but bring no end to the disease itself.

Scientists and the NBCC need to work together to reorder priorities and change the conversation and culture of science. Trust is not lost when advocates call for a deadline and provide a blueprint for meeting it. But it is eroded when scientific infrastructure is unaccountable to the people intended to benefit from its output; when there is not enough emphasis on translating research discoveries to the clinic; and when published results cannot be replicated and marginal advances are overhyped. Meanwhile, more and more people lose their lives.

Take a calculated risk with us. Let's reach for what might in fact be possible.

Frances Visco National Breast Cancer Coalition, Washington DC, USA.

fmvisco@

breastcancerdeadline2020.org

\section{Cancer goal: vaccine is cause for optimism}

As the originator of the project to develop a preventive breastcancer vaccine, which is supported by the US National Breast Cancer Coalition's (NBCC) 2020 deadline, I question your dismissal of this new approach (Nature 491, 637; 2012).

You base your pessimism on the genomic complexity of tumours and the length of time that clinical trials would take to test such a vaccine. But the mature tumours is not relevant for developing a preventive vaccine: the immune system needs only to be preactivated with antigens presented by the nascent tumour. Unstable RNA processing in a tumour is likely to be a richer source of these antigens than genomic mutations.

It could indeed take a decade or more to validate the treatment using today's clinical standards. But trials could be made shorter and less expensive if, for example, there were definitive ways to detect very early tumours.

You say that "discovery does not answer to deadlines", but accomplishment can. Given the alternatives, we should embrace this effort.

Stephen Albert Johnston genomic complexity apparent in
Arizona State University, Tempe, USA.

stephen.johnston@asu.edu

\section{Tie carbon emissions to consumers}

Global carbon dioxide emissions soared from 22.7 billion tonnes in 1990 to 33.9 billion tonnes last year, despite 20 years of attempted mitigation (Nature 491, 656-658; 2012). The sizeable economic gaps between nations are largely responsible for the international deadlock in climate negotiations. A radically new approach is needed.

One solution would be to allocate common but differentiated responsibility for mitigating emissions to individual consumption activities, rather than to countries. Profligate consumers from both developed and emerging countries are the worst offenders for generating non-essential emissions. They should be held accountable for those emissions, irrespective of where they are generated.

Emissions produced after reallocating responsibility in this way could be quantified for nations, lifestyles or even every product consumed. This could be achieved by integrating the 'top-down' consumption-based accounting methods that are used to determine national total consumption emissions with 'bottom-up' carbon-footprint calculations based on analysis of products' life cycles (G. P. Peters Curr. Opin. Environ. Sustain. 2, 245-250; 2010). A living standard, together with a per capita emission quota, would be defined so that people worldwide could meet their basic living requirements without mitigation costs.

Such standardized measures would allow new cap-and-trade policies and carbon-taxing mechanisms to run smoothly and effectively across different consumption groups at a global scale (see M. Grubb Nature 491, 666-667; 2012). 